\title{
Inactivation of medial prefrontal cortex impairs time interval discrimination in rats
}

\author{
Jieun Kim', Amy Hyeyun Jung ${ }^{2}$, Jayoung Byun', Suhyun Jo' and Min Whan Jung ${ }^{1 *}$ \\ ' Neuroscience Laboratory, Institute for Medical Sciences, Ajou University School of Medicine, Suwon, Korea \\ 2 Seoul International School, Seongnam, Korea
}

Edited by:

Jeansok J. Kim, University of

Washington, USA

Reviewed by:

Mark Laubach, Yale University School

of Medicine, USA

Bruno Poucet, CNRS and Université de

Provence, France

Marcelo Caetano,

Brown University, USA

*Correspondence:

Min Whan Jung, Neuroscience

Laboratory, Institute for Medical

Sciences, Ajou University School of

Medicine, Suwon 443-721, Korea.

e-mail:min@ajou.ac.kr

\begin{abstract}
Several lines of evidence suggest the involvement of prefrontal cortex in time interval estimation. The underlying neural processes are poorly understood, however, in part because of the paucity of physiological studies. The goal of this study was to establish an interval timing task for physiological recordings in rats, and test the requirement of intact medial prefrontal cortex (mPFC) for performing the task. We established a temporal bisection procedure using six different time intervals ranging from 3018 to $4784 \mathrm{~ms}$ that needed to be discriminated as either long or short. Bilateral infusions of muscimol (GABA receptor agonist) into the mPFC significantly impaired animal's performance in this task, even when the animals were required to discriminate between only the longest and shortest time intervals. These results show the requirement of intact $\mathrm{mPFC}$ in rats for time interval discrimination in the range of a few seconds.
\end{abstract}

Keywords: interval timing, temporal bisection, muscimol, prefrontal cortex

\section{INTRODUCTION}

The ability to estimate time intervals is critical for survival in many animal species including humans. Results from previous studies suggest the involvement of prefrontal cortex (PFC) in interval timing. In humans, brain imaging studies have shown enhanced blood oxygenation level-dependent signals in the PFC during various timing tasks (Rubia et al., 1998; Rao et al., 2001; Lewis and Miall, 2003; Coull et al., 2004; Hinton and Meck, 2004; Pouthas et al., 2005; Stevens et al., 2007), and focal PFC lesions (Mangels et al., 1998) or transcranial magnetic stimulation of the PFC (Koch et al., 2003; Jones et al., 2004) induces abnormal interval-timing behavior. In animals, lesions to (or inactivation of) the PFC impair interval timing-related behavior in rats (Dietrich et al., 1997; Dietrich and Allen, 1998), cats (Rosenkilde and Divac, 1976) and monkeys (Glickstein et al., 1964; Onoe et al., 2001). Also, physiological studies in monkeys have found PFC neurons that slowly increase their firing rates ("ramping activity") during the delay period of a working memory task (Niki and Watanabe, 1979; Kojima and Goldman-Rakic, 1982; Quintana and Fuster, 1999; Rainer et al., 1999; Brody et al., 2003), which suggests that the PFC might keep track of elapsed time based on slowly changing neuronal activity. Although the exact role of the PFC in time interval discrimination is currently under debate (clock vs. other functions such as working memory and attentional control; e.g., Mangels et al., 1998; Tregellas et al., 2006; Livesey et al., 2007), it is well-agreed that the PFC is a part of the neural system that enables the animal to estimate time intervals and use this information for adaptive control of behavior.

Currently, the neural basis of time perception in the range of seconds to minutes (interval timing; Buhusi and Meck, 2005) is unclear. One major reason is the paucity of physiological studies. Compared to other functions, such as spatial perception, the number of physiological studies examining activity of single neurons related to interval timing (e.g., Pang et al., 2001; Leon and Shadlen, 2003; Matell et al., 2003; Janssen and Shadlen, 2005; Lebedev et al., 2008; Genovesio et al., 2009; Mita et al., 2009) is exceedingly small. More studies examining neural activity in the brain areas critically involved in interval timing while animals are actively engaged in time interval estimation are needed to better understand underlying neural processes. The goal of this study was to establish an interval timing task for physiological recordings in rats, and test whether the intact medial PFC ( $\mathrm{mPFC}$ ) is required for correctly performing the task. A temporal bisection task (Church and Deluty, 1977; Gallistel, 1990) would be useful to this end, particularly for investigating neural processes underlying perceptual timing (Coull and Nobre, 2008). The durations of time intervals used for rats in this task are relatively long (seconds) and animals can perform a large number of trials $(>100)$ so that a sufficient number of spikes can be collected for analysis. Also, interval durations can be titrated so that animals commit a substantial number of errors. This will facilitate finding specific patterns of neural activity that are associated with successful (as opposed to erroneous) discrimination of time intervals, and allow direct comparisons between neurometric and psychometric functions (e.g., Leon and Shadlen, 2003). Finally, compared to the peak procedure which has been used widely to study interval timing behavior of rats (Gallistel, 1990), animals do not make time-dependent behavioral responses in the temporal bisection procedure, alleviating potential compounding effects of behavior on time-dependent neural activity. These features of the temporal bisection task would be useful for physiological studies.

An additional requirement for a behavioral task designed for physiological studies is the minimization of behavioral variations that might influence neural activity. In temporal bisection tasks for rats, animals are typically trained to press either of two levers in a Skinner box (Gallistel, 1990). Under these settings, animal's body 
position and/or orientation during sample interval presentation might vary considerably depending on the previous choice of the animal. There are several different ways of alleviating this problem, and one way is to force the animal to navigate to a central location along a common path after making a choice, which was employed in the present study. We established a temporal bisection procedure in which rats had to navigate to make a choice and to initiate a new trial on a figure 8-shaped maze, and found that inactivation of the $\mathrm{mPFC}$ impairs animal's performance in the task.

\section{MATERIALS AND METHODS SUBJECTS}

Experiments were performed with six young male Sprague-Dawley rats ( $~ 8$-weeks old, $250-270 \mathrm{~g}$ ). Animals were housed individually on a reversed 12/12-h light/dark schedule with light on at 9:00 PM. Experiments were performed twice daily approximately between 10:00 AM and noon and between 6:00 PM and 8:00 PM. The experimental protocol was approved by the Ethics Review Committee for Animal Experimentation of the Ajou University School of Medicine.

\section{BEHAVIORAL TASK}

Animals were trained to perform a temporal bisection task on a figure 8-shaped maze $(63 \times 69 \mathrm{~cm}$, elevated $30 \mathrm{~cm}$ from the floor; 8 -cm-wide track with $3 \mathrm{~cm}$ walls around the track except the central connecting bridge; Figure 1). Each trial began as the animal came back from either goal location (white circles, Figure 1) to the central arm of the maze and broke the central photobeam (arrow, Figure 1) that was placed $3 \mathrm{~cm}$ away from the central bridge. The breaking of the photobeam produced an auditory signal $(3.3 \mathrm{KHz}, 200 \mathrm{~ms}$, $90 \mathrm{db}$ ) that signaled the beginning of a sample time interval. The end of a given time interval was signaled by lowering of the central bridge that allowed the animal to navigate to either goal location. The central bridge was raised when the animal reached either goal location, which was signaled by two photobeams placed $3 \mathrm{~cm}$ away from the water-delivery nozzles (arrows, Figure 1). The animal was required to return to the central arm via the lateral alley.

One of six different time intervals, that were spaced evenly in logarithmic scale, was presented to the animal in each trial, and the animal had to navigate to one designated goal (left or right depending on animal) when a short (3018, 3310 or $3629 \mathrm{~ms})$ interval was presented, and navigate to the opposite goal when a long $(3979,4363$ or $4784 \mathrm{~ms})$ interval was presented in order to obtain water reward $(30 \mu \mathrm{l})$. Time intervals were presented in two different ways in this study. In the random-trial task, six time intervals were presented in equal probability for a total of 180-240 trials (30-40 trials for each interval) per session in random order. In the block-trial task, three pairs of intervals with varying levels of difficulty (easy: 3018 vs. 4784 ms; intermediate: 3310 vs. 4363 ms; hard: 3629 vs. $3979 \mathrm{~ms}$ ) were presented in blocks of 60 trials (a total of 180 trials per session), with the sequence of time intervals within a block randomized ( 30 trials per each interval). The presentation of time intervals, delivery of water and raising/lowering of the central bridge were automatically controlled by a personal computer using LabView software (National Instruments, Austin, TX, USA).

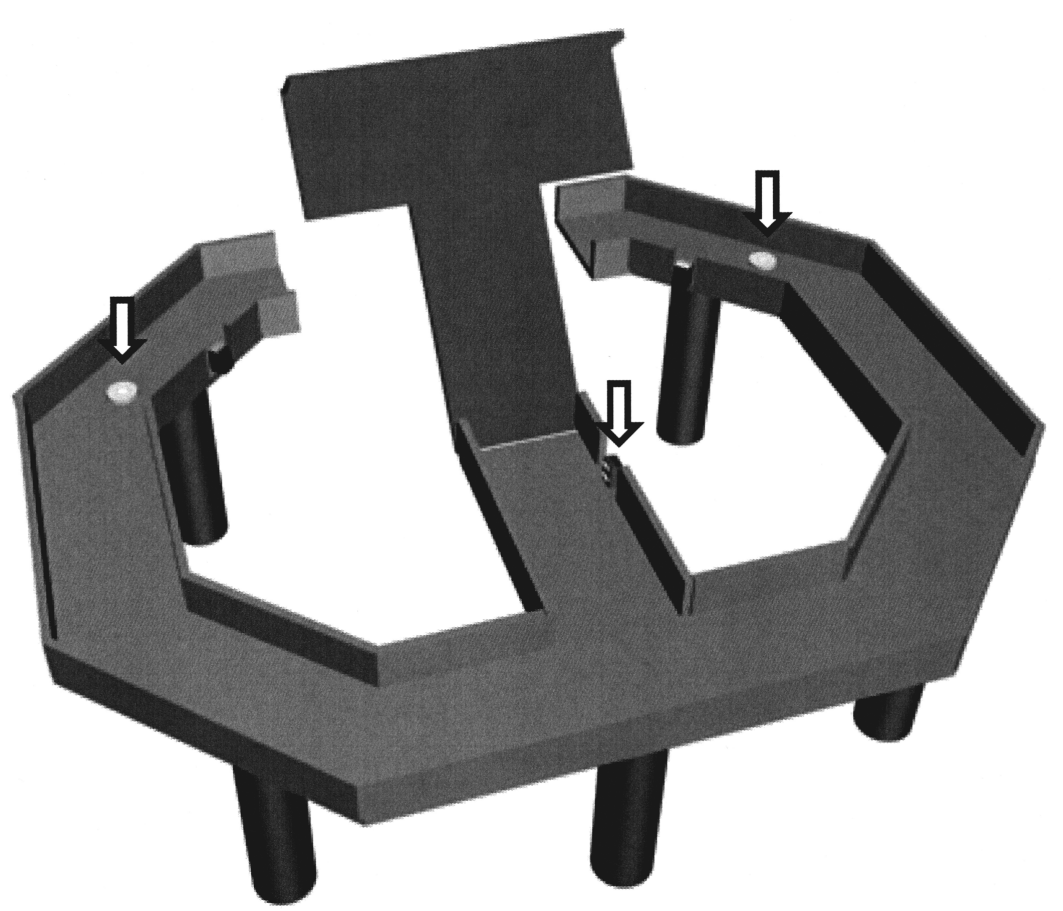

FIGURE 1 | Behavioral task. Rats were tested on a figure 8-shaped maze to choose between two target locations (white circles) depending on the duration of a given time interval. A time interval began when the animal broke the central photobeam (arrow). The central connecting bridge was lowered at the end of the time interval allowing the animal to proceed to either goal location. The central bridge was elevated again when the animal broke a photobeam (arrow) at either goal location. Scale bar: $10 \mathrm{~cm}$. 


\section{TRAINING PROCEDURES \\ Shaping}

The time line of training and testing procedures is summarized in Figure 2. Water deprivation and maze adaptation began following 1 week of extensive handling. Animals experienced one shaping session per day approximately between 10:00 AM and noon. Each animal was allowed to freely explore the maze for $\sim 30$ min without water reward on the first day, and, on the second day, guided to navigate in the correct direction (central arm $\rightarrow$ goal $\rightarrow$ lateral alley $\rightarrow$ central arm) by providing water drops on the maze floor for $\sim 30 \mathrm{~min}$. On day 3, water was delivered through the water-delivery nozzle (white circles, Figure 1) when animals navigated to either goal location in the correct direction for the training duration of $\sim 1 \mathrm{~h}$. Water drops were provided to the animals as necessary when they were not willing to run toward a goal. The same training was repeated on day 4 , but with the central bridge raised when the animals arrived at either goal location, and lowered immediately when they returned to the central arm and broke the central photobeam.

\section{Training}

Animals were trained twice daily in most cases. Pre-training began with the shortest interval $(3018 \mathrm{~ms})$. Only the shortest interval was given in each trial and the animals were rewarded with water when they navigated to the correct goal. The training continued until they chose the correct goal $>80 \%$ (a total of $\sim 60$ trials and $\sim 1$-h training per session; $\sim 2$ days). Next, only the longest interval (4784 ms) was given in each trial and the animals were trained to navigate to the opposite goal until they chose it $>80 \%$ ( $~ 60$ trials and $\sim 1-\mathrm{h}$ training per session; $\sim 2$ days). Then the shortest and longest intervals (3018 and $4784 \mathrm{~ms}$ ) were alternatively provided in blocks of trials for $\sim 1 \mathrm{~h}$ per session. The animals were initially trained with 3 blocks (in the sequence of 3018-4784-3018 ms) of 50 trials, but the number of blocks was gradually increased up to 15 while the number of trials in each block was gradually reduced down to 10 over the course of $\sim 1$ week of training. When the proportion of correct choices reached $>80 \%$, the same numbers of the shortest and longest intervals were presented in random order for $\sim 1$ week $(\sim 1$-h training per session). When the animal's performance was $>90 \%$ correct, two intermediate intervals (3310 and $4363 \mathrm{~ms}$ ) were added to the training procedure. The animals were presented with the shortest and longest intervals (3018 and $4784 \mathrm{~ms}$ ) in random order in the first block (90 trials), and then with the two intermediate intervals (3310 and $4363 \mathrm{~ms}$ ) in random order in the second block (90 trials) for $\sim 4$ days ( $\sim 1$-h training per session). When the animal chose correct targets $>80 \%$ of trials in the second block, the remaining two intervals (3629 and $3979 \mathrm{~ms}$ ) were introduced. Three blocks of interval pairs were presented in the sequence of easy (3018 and $4784 \mathrm{~ms}$ ), intermediate (3310 and $4363 \mathrm{~ms}$ ) and hard pairs (3629 and $3979 \mathrm{~ms}$ ) for $\sim 4$ days ( $\sim 1$-h training per session). Two intervals were presented in random order with equal frequency within each block, and the number of trials in each block was gradually increased so that the total number of trials became 180 (60 trials per block). Pre-training ended when the animal correctly discriminated the difficult interval pair in $>65 \%$ of trials. Overall, the animals went through 50-56 sessions (25-28 days) of pre-training up to this stage, and their performance during the final session was $74.9 \pm 2.3 \%$ correct choices in the difficult block and $84.5 \pm 1.4 \%$ correct choices for the entire session ( $n=6$ animals).

The three rats used in the drug injection experiment (rat \#4-6; experiment 2) were subject to surgery at this stage. The other three rats were further trained in the random-trial task (rat \#1-3; experiment 1). In each session, they were first trained with the easy pair (3018 or $4784 \mathrm{~ms}$ ) for a total of 20 trials (practice block), and then trained with randomly presented six time intervals for a total of 180-240 trials for $\sim 7$ days. The training phase ended when the animal correctly discriminated the difficult interval samples (3629

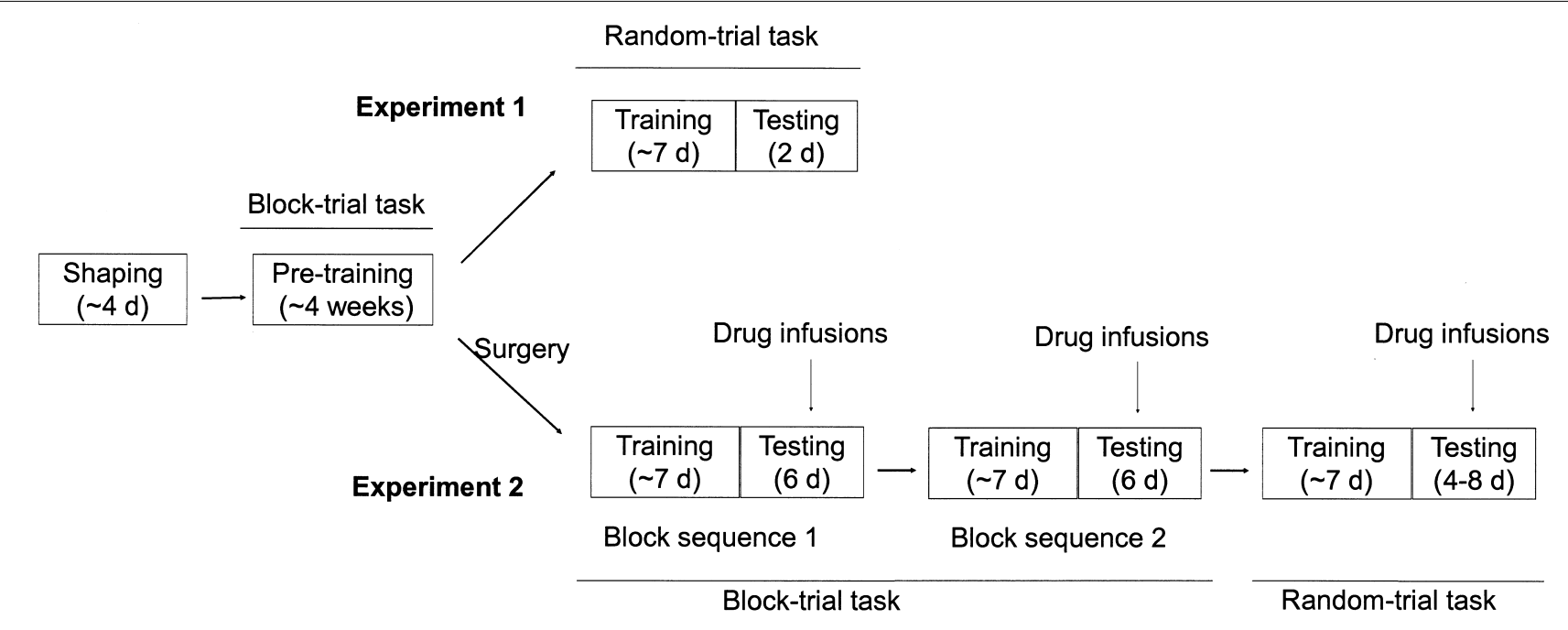

FIGURE 2 |Training and testing procedures. Animals went through shaping ( $\sim 4$ days) and pre-training ( $\sim 4$ weeks) in the block-trial task. One group of animals was further trained to perform the random-trial task $(\sim 7$ days) and their performance was tested (experiment 1). The other group of animals was subject to surgery, re-trained in the block-trial task, and tested in the same task with saline, $0.1 \mu \mathrm{g}$ muscimol, or $0.2 \mu \mathrm{g}$ muscimol infused into the mPFC. They were further trained in the trial-block task with a different block sequence, and tested under drug treatment. Finally, these animals were trained in the random-trial task ( $\sim 7$ days) and their performance was tested under drug treatment (experiment 2). 
and $3979 \mathrm{~ms}$ ) in $>65 \%$ of trials (animal's performance in the last training session: $68.4 \pm 3.4$ and $82.4 \pm 2.2 \%$ correct choices for the difficult and all interval samples, respectively). Choice data were then collected for four sessions using the same procedure ( 240 trials following 20 practice trials at the outset).

\section{TESTING WITH DRUG INFUSIONS}

In all post-surgery training and testing sessions (experiment 2), the animals were first trained with the easy interval pair (3018 vs. $4784 \mathrm{~ms}$ ) for a total of 20 trials (practice block) before beginning the main training/testing sessions (two sessions per day). Following 3 days of recovery from surgery, the animals were re-trained with the block-trial procedure in the sequence of easy, intermediate and hard pairs for a total of 180 trials per session for $\sim 1$ week. Drug infusions began when they reached a criterion performance at each level of difficulty (80,70 and $60 \%$ correct choices for easy, intermediate and hard pairs, respectively; animal's performance in the final training session: $92.3 \pm 3.1$, $85.3 \pm 2.5$, and $69.2 \pm 4.8 \%$ correct choices for the easy, intermediate and hard pairs, respectively, and $82.3 \pm 1.9 \%$ correct choices for the entire session). Animals were tested in the same block sequence with saline (S), $0.1 \mu \mathrm{g}$ muscimol (M1), or $0.2 \mu \mathrm{g}$ muscimol (M2) infused bilaterally for the next 6 days in the order of S-M1-M1-S-M2-M2. The animals were then trained with a different sequence of interval pairs (rat \#4: hard-easy-intermediate; rat \#5: intermediate-easy-hard; rat \#6: intermediate-hard-easy) until they reached the criterion $(80,70$ and $60 \%$ correct choices for easy, intermediate and hard pairs, respectively) without drug infusions for $\sim 1$ week (animal's performance in the last session: $89.6 \pm 3.2,77.8 \pm 3.9,65.0 \pm 1.0 \%$ correct choices for the easy, intermediate and hard pairs, respectively, and $77.5 \pm 1.8 \%$ correct choices for the entire session), and tested in the same sequence of interval pairs (180 trials per session) with either saline or muscimol infused bilaterally in the same manner for 6 days (S-M1-M1-S-M2-M2).

At the completion of the block-trial task, the animals were trained in the random-trial task until their performance reached $>65 \%$ correct choices for the difficult interval samples (3629 and $3979 \mathrm{~ms}$ ) for a total of 180 trials per session $(\sim 1$ week of training; animal's performance in the last training session: $69.1 \pm 2.4$ and $84.5 \pm 0.3 \%$ correct choices for the difficult and all interval samples, respectively). Animals were tested with either saline or muscimol infused bilaterally for 4-8 days (rat \#4: S-M1-M1-S-M2-M2; rat \#5: S-M1-M1-S; rat\#6:S-M1-M1-M1-S-M2-M2-M2).All drug infusion experiments were performed once per day approximately between 10:00 AM and noon, and animals went through an additional training session in the afternoon (approximately 8:00-10:00 PM).

\section{SURGERY}

Animals were deeply anesthetized via intraperitoneal injection of $50 \mathrm{mg} / \mathrm{kg}$ sodium pentobarbital and mounted on a stereotaxic frame. Small burr holes were drilled on the skull to remove bones over the infusion sites, and 26-gauge guide cannulae were implanted bilaterally into the mPFC $(3.20 \mathrm{~mm}$ anterior, $2.21 \mathrm{~mm}$ lateral, $3.26 \mathrm{~mm}$ ventral to bregma) at an angle $20^{\circ}$ from the midline. Five anchor screws were placed in the skull and the implanted cannulae were secured in place with dental acrylic.

\section{DRUG INFUSION}

Buffered-saline $(0.9 \%, \mathrm{pH} \sim 7.4)$ or muscimol (Tocris Cookson, Bristol, UK) dissolved in $0.9 \%$ buffered-saline was micro-infused bilaterally into the mPFC through an inner cannula (33 gauge) that extended $1 \mathrm{~mm}$ below the guide cannula. The inner cannula was connected by a polyethylene tube (PE-20) to a $10-\mu \mathrm{l}$ Hamilton microsyringe, that was driven by a microsyringe pump (KD Scientific Inc., USA; model: KDS100). 0.1 0.2 $\mu$ l of saline or muscimol solution (concentration: $1 \mu \mathrm{g} / \mu \mathrm{l}$, speed: $10 \mu \mathrm{l} / \mathrm{h}$ ) was delivered to each hemisphere and the cannula remained in place for $1 \mathrm{~min}$. Behavioral testing started $30 \mathrm{~min}$ after the completion of drug infusions.

\section{HISTOLOGY}

At the completion of behavioral testing, animals were overdosed with sodium pentobarbital and perfused intracardially with $0.9 \%$ saline followed by $10 \%$ buffered-formalin. The brain was removed, left in $10 \%$ buffered-formalin, and transferred to a $30 \%$ sucrose solution for 2 days until it sank to the bottom. Forty micron coronal sections were cut on a sliding microtome and stained with cresyl violet to verify cannula placements.

\section{DATA ANALYSIS \\ Block-trial task}

Animal's choice data were converted to \% correct choice (choice accuracy) for each block (easy: 3018 vs. $4784 \mathrm{~ms}$; intermediate: 3310 vs. 4363 ms; hard: 3629 vs. 3979 ms), averaged across all sessions of the same drug treatment (saline, $0.1 \mu \mathrm{g}$ muscimol or $0.2 \mu \mathrm{g}$ muscimol infusions) for each animal, and subjected to two-way ANOVA with two repeated measures (drug and bock).

\section{Random-trial task}

Animal's choice data were averaged across all sessions of the same type for each animal and subjected to the following logistic regression:

$\log \left(\frac{P_{\text {long }}}{1-P_{\text {long }}}\right)=a+b T$,

where $P_{\text {long }}$ is the proportion of long-choice trials, $T$ denotes the sample interval duration, and $a$ and $b$ are constants. The bisection point (the sample duration corresponding to $P_{\text {long }}=0.5$ ) was determined as a measure of the point of subjective equality (PSE), and the slope at the bisection point $\left(S_{\mathrm{B}}\right)$ was calculated as a measure of sensitivity to sample duration as the following:

$S_{\mathrm{B}}=\frac{b}{4}$.

Note that our procedure is different from the conventional temporal bisection procedure in that correctly discriminated sample intervals were always rewarded in our study whereas only the anchor intervals (the longest and shorest intervals) are rewarded in the conventional procedure (Church and Deluty, 1977; Gallistel, 1990). Thus, the PSE determined in our study is not equivalent to the conventional PSE which provides information on the "perceived middle" between the longest and shortest intervals. 
Statistical significance of muscimol effects on choice accuracy, the bisection point, and $S_{\mathrm{B}}$ was tested with one-way ANOVA (experiment 2). Student's t-test (two-tailed) was used to compare these measures between non-operated animals (experiment 1) and saline-infused animals (experiment 2).

\section{Choice strategy}

We also examined whether muscimol infusions altered choice strategy of the animals. In order to test potential muscimol effects on perseverative behavior, the degree of choice bias [the percentage of preferred choices in a given block (block-trial task) or session (random-trial task)] and the proportion of repeated choices (ranging from 2 to 4 ) were examined. Also, to test whether the animals simply followed the win-stay-loose-switch strategy irrespective of the duration of a given sample interval, the proportions of win-stay (repeating the rewarded choice) and loose-switch choices (alternating from the unrewarded choice) were examined. Finally, to test whether muscimol infusions affected locomotive functions of the animal, response latency (the duration between the offset of a sample interval and animal's arrival at a goal location) was compared. These behavioral variables during the block-trial task were subjected to two-way ANOVA (with drug and block as two repeated measures) and those during the random-trial task were analyzed with one-way ANOVA.
All data are expressed as mean \pm SEM. A $p$ value $<0.05$ was used as the criterion for a significant statistical difference.

\section{RESULTS \\ EXPERIMENT 1: PERFORMANCE IN THE RANDOM-TRIAL TASK}

The probability to choose the long-target increased monotonically as a function of sample duration (Figure 3 ). The relationship between the probability to choose the long-target and sample duration was well accounted for by the logistic regression in each animal $\left(R^{2}=0.995 \pm 0.002\right)$. Values for choice accuracy, the bisection point and the slope at the bisection point $\left(S_{\mathrm{B}}\right)$ are summarized in Table 1.

\section{EXPERIMENT 2: EFFECTS OF MPFC INACTIVATION ON TIME INTERVAL DISCRIMINATION Cannula placements}

All operated animals had guide cannulae placed within the border of the mPFC (prelimbic and infralimbic areas) bilaterally (Figure 4).

\section{Block-trial task}

Choice accuracy. Infusions of muscimol into the mPFC lowered the proportion of correct sample interval discrimination in a dose-dependent manner (Figure 5A). Two-way ANOVA revealed
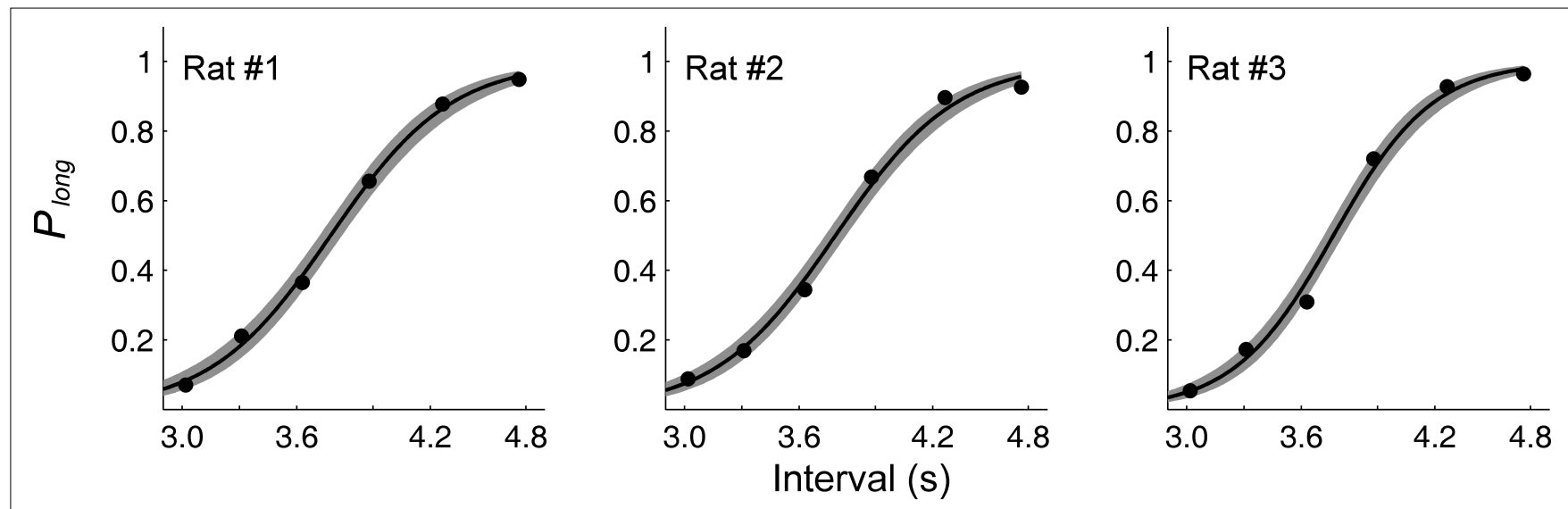

FIGURE 3 | Choice behavior in the random-trial task. The graphs show the proportion of long-target choices $\left(P_{\text {long }}\right)$ as a function of sample duration across four behavioral sessions for each non-operated animal (experiment 1). The solid lines were determined by logistic regression and the shading indicates $95 \%$ confidence interval.

Table 1 |Animal's performance in the random-trial task. Results are expressed as mean \pm SEM ( $n=3$ animals in each experiment).

\begin{tabular}{|c|c|c|c|c|}
\hline \multicolumn{5}{|l|}{ EXP 1: } \\
\hline Bisection point (s) & $3.78 \pm 0.01$ & & & \\
\hline Slope $\left(S_{B}\right)$ & $0.85 \pm 0.05$ & & & \\
\hline \multicolumn{5}{|l|}{ EXP 2: } \\
\hline Bisection point (s) & & $3.82 \pm 0.03$ & $3.97 \pm 0.17$ & $3.87 \pm 0.43$ \\
\hline Slope $\left(S_{B}\right)$ & & $0.77 \pm 0.07$ & $0.34 \pm 0.10$ & $0.29 \pm 0.03$ \\
\hline
\end{tabular}




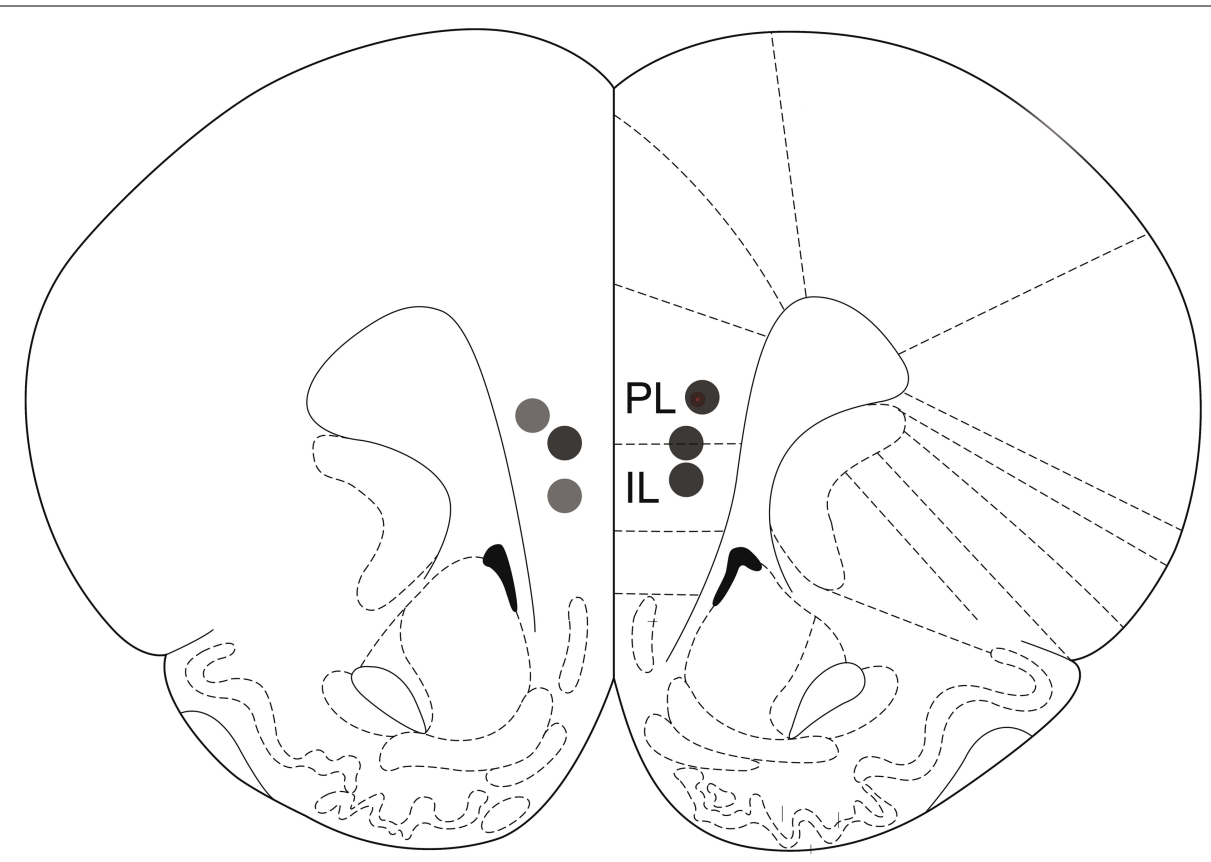

FIGURE 4 | Drug infusion sites. Filled circles indicate locations where infusion cannula tips were placed. PL: prelimbic cortex; IL: infralimbic cortex. Coronal brain section templates are from Paxinos and Watson (1998).

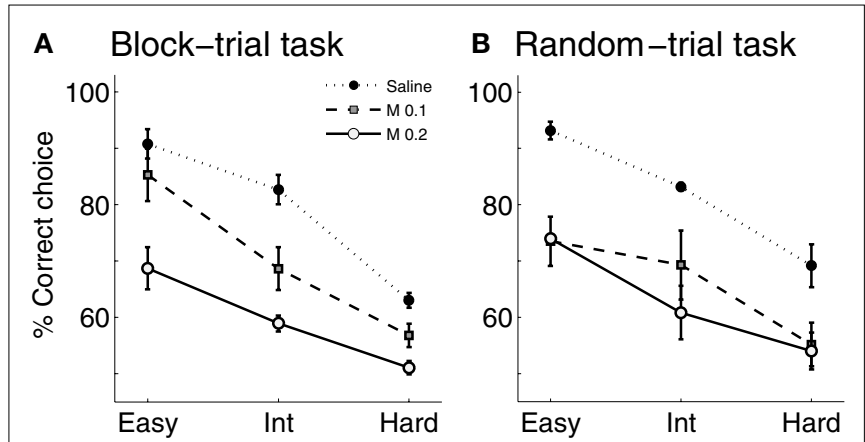

FIGURE 5 | Effect of mPFC inactivation on time interval discrimination. The graphs show the proportion of correct choices at different levels of difficulty (easy, intermediate and hard) following saline or muscimol infusions (experiment 2). (A) Block-trial task. (B) Random-trial task. Error bars indicate SEM ( $n=3$ animals in each task). Int: intermediate level; M 0.1: muscimol $0.1 \mu \mathrm{g}$ infusions; M 0.2: muscimol $0.2 \mu \mathrm{g}$ infusions.

significant main effects of $\operatorname{drug}\left(F_{(2,4)}=76.48, p=0.001\right)$ and block $\left(F_{(2,4)}=37.49, p=0.003\right)$, but not significant drug $\times$ block interaction $\left(F_{(4,8)}=1.32, p=0.341\right)$. Separate examination of the behavioral data during the easy block (3018 vs. $4784 \mathrm{~ms}$ intervals) showed that muscimol infusions significantly lowered choice accuracy even between the longest and shortest intervals (one-way repeated measure ANOVA, $\left.F_{(2,4)}=11.07 ; p=0.023\right)$.

Choice strategy. Values of the choice strategy-related variables are summarized in Table 2. Muscimol infusions had no significant effect on the degree of choice bias (main effect of drug: $F_{(2,4)}=1.23, p=0.384$; main effect of block: $F_{(2,4)}=0.26, p=0.781$; effect of drug $\times$ block interaction: $\left.F_{(4,8)}=0.71, p=0.606\right)$, the proportion of repeated choices (two consecutive choices, main effect of drug: $F_{(2,4)}=0.00, p=1.000$; main effect of block: $F_{(2,4)}=0.11, p=0.897$; effect of drug $\times$ block interaction: $F_{(4,8)}=0.36, p=0.829$; three consecutive choices, main effect of drug: $F_{(2,4)}=0.97, p=0.453$; main effect of block: $F_{(2,4)}=1.03$, $p=0.436$; effect of drug $\times$ block interaction: $F_{(4,8)}=0.16, p=0.952$; four consecutive choices, main effect of drug: $F_{(2,4)}=2.22, p=0.526$; main effect of block: $F_{(2,4)}=4.47, p=0.095$; effect of drug $\times$ block interaction: $\left.F_{(4,8)}=0.36, p=0.828\right)$, the proportion of win-stay choices (main effect of drug: $F_{(2,4)}=0.64, p=0.575$; main effect of block: $F_{(2,4)}=11.86, p=0.021$; effect of drug $\times$ block interaction: $F_{(4,8)}=2.28, p=0.149$ ), the proportion of loose-switch choices (main effect of drug: $F_{(2,4)}=0.04, p=0.962$; main effect of block: $F_{(2,4)}=0.26$, $p=0.785$; effect of drug $\times$ block interaction: $\left.F_{(4,8)}=0.24, p=0.908\right)$, or the response latency (main effect of drug: $F_{(2,4)}=8.33, p=0.102$; main effect of block: $F_{(2,4)}=0.39, p=0.601$; effect of drug $\times$ block interaction: $F_{(4,8)}=1.08, p=0.420$; Greenhouse-Geisser corrected). As described, the only significant effect was the main effect of block on the proportion of win-stay choices (easyblock: $43.1 \pm 2.2 \%$; intermediate block: $49.2 \pm 1.5 \%$; hard block: $51.9 \pm 1.9 \%$ ). It is unclear why animals tended to show less frequent win-stay choices in easier blocks.

\section{Random-trial task}

Choice accuracy and time interval sensitivity. Choice behavior of saline-infused animals was similar to that of non-operated animals in the experiment 1 (unpaired $t$-test, the proportion of correct choices: $t_{(34)}=0.16, p=0.877$; bisection point: $t_{(2.1)}=1.230$, $p=0.338 ; S_{\mathrm{B}}: t_{(4)}=0.874, p=0.432$; Figures 3 and 6; Table 1), and it was well accounted for by the logistic regression $\left(R^{2}=0.973 \pm 0.010\right)$. Muscimol infusions lowered choice accuracy (Figure 5B) and flattened the interval-choice relationship considerably (logistic 
Table 2 | Behavioral measures related to choice strategy. The degree of choice bias, the proportion of consecutive same choices (repetition; 2-4), the proportions of win-stay and loose-switch choices, and the response latency are summarized for the block-trial and random-trial tasks (mean \pm SEM; $n=3$ animals in each task). E, I and H: easy, intermediate and hard blocks, respectively.

\begin{tabular}{|c|c|c|c|c|c|c|c|c|c|}
\hline \multirow[b]{2}{*}{ Block } & \multicolumn{3}{|c|}{ Saline } & \multicolumn{3}{|c|}{ Muscimol $(0.1 \mu \mathrm{g})$} & \multicolumn{3}{|c|}{ Muscimol $(0.2 \mu \mathrm{g})$} \\
\hline & $\mathbf{E}$ & $\mathbf{I}$ & $\mathbf{H}$ & $\mathbf{E}$ & I & $\mathbf{H}$ & $\mathbf{E}$ & I & $\mathbf{H}$ \\
\hline Choice bias (\%) & $50.0 \pm 0.1$ & $50.0 \pm 0.0$ & $50.0 \pm 0.0$ & $50.5 \pm 1.1$ & $50.4 \pm 0.5$ & $50.4 \pm 0.5$ & $50.0 \pm 0.0$ & $50.0 \pm 0.0$ & $50.2 \pm 0.2$ \\
\hline Repetition $(2 \times, \%)$ & $44.5 \pm 1.2$ & $47.0 \pm 0.4$ & $45.3 \pm 1.3$ & $46.3 \pm 1.7$ & $45.4 \pm 3.6$ & $43.9 \pm 3.8$ & $46.1 \pm 5.2$ & $45.7 \pm 7.1$ & $45.9 \pm 10.9$ \\
\hline Repetition $(3 \times, \%)$ & $17.4 \pm 0.6$ & $19.1 \pm 1.3$ & $20.6 \pm 1.7$ & $18.7 \pm 2.7$ & $20.4 \pm 4.4$ & $21.0 \pm 4.0$ & $25.0 \pm 5.6$ & $23.1 \pm 6.6$ & $26.6 \pm 10.1$ \\
\hline Win-stay (\%) & $46.0 \pm 1.3$ & $47.8 \pm 1.2$ & $50.2 \pm 2.1$ & $45.8 \pm 0.8$ & $46.6 \pm 3.6$ & $49.1 \pm 2.1$ & $37.5 \pm 5.8$ & $53.1 \pm 0.8$ & $56.4 \pm 4.2$ \\
\hline Loose-switch (\%) & $46.0 \pm 5.3$ & $52.0 \pm 3.2$ & $48.7 \pm 2.3$ & $50.8 \pm 12.0$ & $49.9 \pm 4.3$ & $46.1 \pm 4.0$ & $51.2 \pm 4.4$ & $46.1 \pm 5.1$ & $46.5 \pm 3.9$ \\
\hline Response latency (s) & $0.51 \pm 0.05$ & $0.53 \pm 0.05$ & $0.52 \pm 0.05$ & $0.44 \pm 0.04$ & $0.44 \pm 0.04$ & $0.44 \pm 0.04$ & $0.45 \pm 0.03$ & $0.46 \pm 0.02$ & $0.45 \pm 0.03$ \\
\hline \multicolumn{10}{|c|}{ RANDOM-TRIALTASK } \\
\hline Choice bias (\%) & & $50.1 \pm 0.07$ & & & $50.0 \pm 0.03$ & & & $50.0 \pm 0.0$ & \\
\hline Loose-switch (\%) & & $50.3 \pm 7.9$ & & & $47.3 \pm 7.3$ & & & $47.3 \pm 2.8$ & \\
\hline Response laency (s) & & $0.51 \pm 0.2$ & & & $0.45 \pm 0.12$ & & & $0.45 \pm 0.17$ & \\
\hline
\end{tabular}
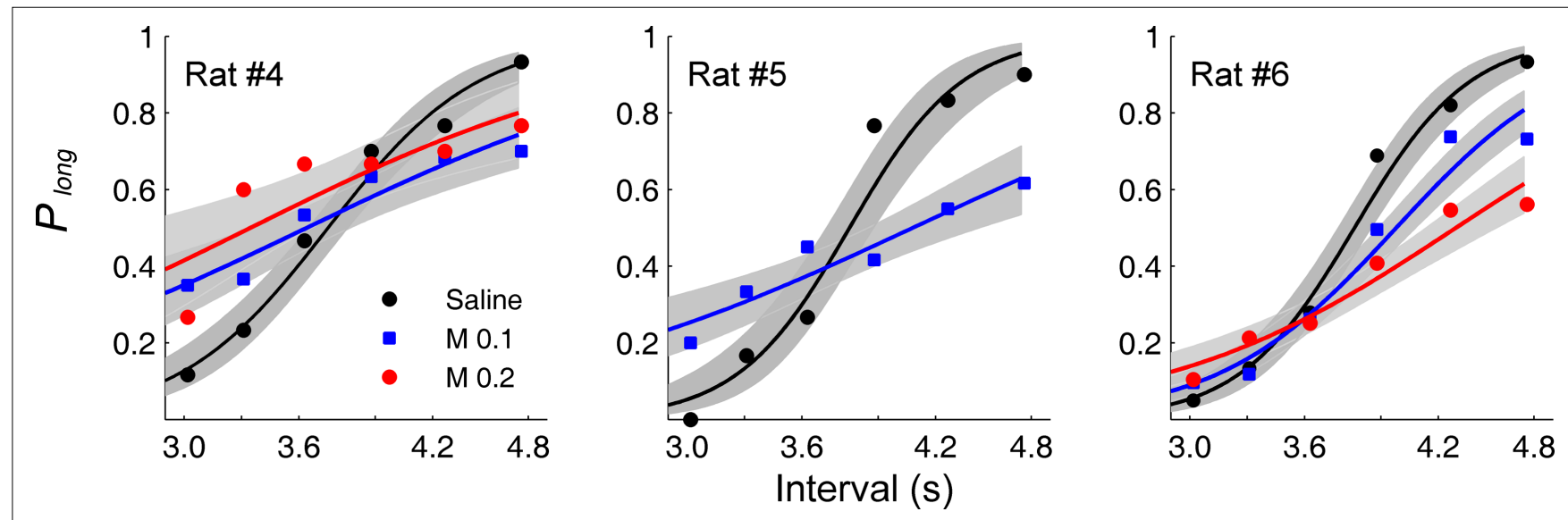

FIGURE 6 | Effect of mPFC inactivation on animal's choice in the random-trial task. The proportion of long-target choices $\left(P_{\text {long }}\right)$ is plotted against sample interval duration under different drug treatment conditions for each animal. The solid lines were determined by logistic regression and the shading indicates $95 \%$ confidence interval. M 0.1: muscimol $0.1 \mu \mathrm{g}$ infusions; M 0.2: muscimol $0.2 \mu \mathrm{g}$ infusions.

regression, $R^{2}=0.927 \pm 0.015$ and $0.821 \pm 0.100$ for 0.1 and $0.2 \mu \mathrm{g}$ muscimol infusions, respectively; Figure 6). Muscimol infusions had no significant effect on the bisection point (one-way ANOVA: $\left.F_{(2,5)}=0.11, p=0.894\right)$, but significantly decreased the proportion of correct choices $\left(F_{(2,5)}=8.28, p=0.025\right)$ and $S_{\mathrm{B}}\left(F_{(2,5)}=10.88\right.$, $p=0.015$; Table 1).

Choice strategy. Muscimol infusions did not have significant effect on the degree of choice bias $\left(F_{(2,5)}=0.98, p=0.438\right)$, the proportion of repeated choices (two consecutive choices, $F_{(2,5)}=0.10, p=0.908$; three consecutive choices, $F_{(2,5)}=0.09, p=0.919$; four consecutive choices; $\left.F_{(2,5)}=0.51, p=0.627\right)$, the proportion of win-stay choices $\left(F_{(2,5)}=0.25, p=0.787\right)$, the proportion of loose-switch choices $\left(F_{(2,5)}=2.90, p=0.146\right)$, or the response latency $\left(F_{(2,5)}=0.33\right.$, $p=0.731$; Table 2).

\section{DISCUSSION}

The first goal of this study was to establish an interval timing task for physiological recordings in rats. To this end, we developed a temporal bisection procedure (random-trial task) that would be 
useful for physiological studies (see Introduction). The second goal was to test whether intact $\mathrm{mPFC}$ is required for performing the task. Timing-related neural data obtained from a brain structure that is critically involved in the employed timing task would be more informative than the data obtained from a brain structure that is dispensable for performing the task. In order to examine the effect of mPFC inactivation under varying levels of difficulty, we tested animals not only in the random-trial task, but also in the block-trial task. Since only two intervals are repeatedly presented in a given block of trials in the block-trial task, the chance for other functions of the PFC to be compounded with interval discrimination would be lower. Consistent impairments in both versions of the task would provide stronger evidence for the role of $\mathrm{mPFC}$ in time interval discrimination in the current task (cf. Tregellas et al., 2006). Indeed, muscimol infusions significantly impaired animal's performance in both versions of the task. It is notable that muscimol-infused animals were impaired even in discriminating the shortest time interval from the longest one (easy block). Moreover, muscimol infusions did not alter the chance to repeat the same choice (perseveration), the degree of win-stay-loose-switch behavior, or the response latency. Collectively, these results provide converging evidence that temporary inactivation of rat mPFC impairs neural processes that are related to time interval discrimination. In a previous study (Dietrich and Allen, 1998), lesions to the mPFC flattened peak-interval (40 s) responses of rats. Our results show that mPFC inactivation impairs time interval discrimination in the range of a few seconds, adding to the existing body of evidence for the role of the PFC in interval timing across different animal species.

It is unclear, however, which aspect of time interval discrimination the $\mathrm{mPFC}$ is in charge of. The $\mathrm{mPFC}$ inactivation might have disrupted the process of estimating elapsed time (i.e., clock function). Alternatively, the mPFC might be important for storing or retrieving into working memory of the information on the reference intervals (bisection point or anchor intervals). It is also possible that the $\mathrm{mPFC}$ contributes to the performance in the current task by providing such executive functions as attentional control (Fuster, 1997), arithmetic operation (computing the bisection point or subtracting the bisection point from a sample interval duration, for example; Dehaene et al., 2004), or rule encoding (Jung et al., 2008). It is unlikely that mPFC inactivation suppressed inhibitory control functions of the mPFC because the animals did not show perseverative behavior. It is also unlikely that the process of loading a sample interval into working memory was the key compromised function of the mPFC in the present task, because the animal was allowed to make a choice immediately after a sample interval was presented. Finally, it is not very likely that the mPFC-inactivated animals were impaired in using task rules, because they did not rely on a simple win-stay-loose-switch strategy ignoring sample durations. To summarize, whereas some functions of the PFC might be excluded from likely contributors, it is unclear exactly which function of the mPFC is the key contributor to the performance of the animal in the present task. Additional studies are required to resolve this issue, and physiological studies might provide particularly valuable information on this matter.

\section{ACKNOWLEDGMENTS}

We thank Drs. Yun Bok Kim and Namjung Huh for their help with data analysis. This work was supported by Korea Science and Engineering Foundation Grant R01-2008-000-10287-0, Korea Research Foundation Grant KRF-2008-314-H00006, and the Cognitive Neuroscience Program of the Korea Ministry of Science and Technology (Min Whan Jung).

\section{REFERENCES}

Brody, C.D.,Hernández,A.,Zainos,A., and Romo, R. (2003). Timing and neural encoding of somatosensory parametric workingmemory in macaque prefrontal cortex. Cereb. Cortex 13, 1196-1207.

Buhusi, C. V., and Meck, W. H. (2005). What makes us tick? Functional and neural mechanisms of interval timing. Nat. Rev. Neurosci. 6, 755-765.

Church, R. M., and Deluty, M. Z. (1977). Bisection of temporal intervals. J. Exp. Psychol. Anim. Behav. Process 3, 216-228.

Coull, J. T., and Nobre, A. C. (2008). Dissociating explicit timing from temporal expectation with fMRI. Curr. Opin. Neurobiol. 18, 137-144.

Coull, J. T., Vidal, F., Nazarian, B., and Macar, F. (2004). Functional anatomy of the attentional modulation of time estimation. Science 303, 1506-1508.

Dehaene, S., Molko, N., Cohen, L., and Wilson, A. J. (2004). Arithmetic and the brain. Curr. Opin. Neurobiol. 14, 218-224.

Dietrich, A., and Allen, J. D. (1998). Functional dissociation of the prefrontal cortex and the hippocampus in timing behavior. Behav. Neurosci. 112, 1043-1047.

Dietrich, A., Frederick, D. L., and Allen, J. D. (1997). The effects of total and subtotal prefrontal cortex lesions on the timing ability of the rat. Psychobiology 25, 191-201.

Fuster, J. M. (1997). The Prefrontal Cortex: Anatomy, Physiology, and Neuropsychology of the Frontal Lobe, 3rd Edn. Philadelphia, Lippincott-Raven.

Gallistel, C. R. (1990). The Organization of Learning. Cambridge, MIT Press.

Genovesio, A., Tsujimoto, S., and Wise, S. P. (2009). Feature- and order-based timing representations in the frontal cortex. Neuron 63, 254-266.

Glickstein, M., Quigley, D. E., and Stebbins, W.C. (1964). Effect of frontal and parietal lesions on timing behaviour in monkeys. Psychonom. Sci. 1, 265-266.

Hinton, S. C., and Meck, W. H. (2004). Frontal-striatal circuitry activated by human peak-interval timing in the supra-seconds range. Brain Res. Cogn. Brain Res. 21, 171-182.
Janssen, P., and Shadlen, M. N. (2005). A representation of the hazard rate of elapsed time in macaque area LIP. Nat. Neurosci. 8, 234-241.

Jones, C. R., Rosenkranz, K., Rothwell, J. C. and Jahanshahi, M. (2004). The right dorsolateral prefrontal cortex is essential in time reproduction: an investigation with repetitive transcranial magnetic stimulation. Exp. Brain Res. 158, 366-372.

Jung, M. W., Baeg, E. H., Kim, M. J., Kim, Y. B., and Kim, J. J. (2008). Plasticity and memory in the prefrontal cortex. Rev. Neurosci. 19, 29-46.

Koch, G., Olivieri, M., and Caltagirone, C. (2003). Underestimation of time perception after repetitive transcranial magnetic stimulation. Neurology 60, 1844-1846.

Kojima, S., and Goldman-Rakic, P. S. (1982). Delay-related activity of prefrontal neurons in rhesus monkeys performing delayed response. Brain Res. 248, 43-49.

Lebedev, M. A., O'Doherty, J. E., and Nicolelis, M. A. (2008). Decoding of temporal intervals from cortical ensemble activity. J. Neurophysiol. 99 , $166-186$.

Leon, M. I., and Shadlen, M. N. (2003). Representation of time by neurons in the posterior parietal cortex of the macaque. Neuron 38, 317-327.

Lewis, P. A., and Miall, R. C. (2003). Brain activation patterns during measurement of sub- and suprasecond intervals. Neuropsychologia 41, 1583-1592.

Livesey, A. C., Wall, M. B., and Smith, A. T (2007). Time perception: manipulation of task difficulty dissociates clock functions from other cognitive demands. Neuropsychologia 45, 321-331.

Mangels, J. A., Ivry, R. B., and Shimizu, N. (1998). Dissociable contributions of the prefrontal and neocerebellar cortex to time perception. Cogn. Brain Res. 7, 15-39.

Matell, M. S., Meck, W. H., and Nicolelis, M.A. (2003). Interval timing and the encoding of signal duration by ensembles of cortical and striatal neurons. Behav. Neurosci. 117, 760-773.

Mita, A., Mushiake, H., Shima, K., Matsuzaka, Y., and Tanji, J. (2009). 
Interval time coding by neurons in the presupplementary and supplementary motor areas. Nat. Neurosci. 12, 502-507.

Niki, H., and Watanabe, M. (1979). Prefrontal and cingulate unit activity during timing behavior in the monkey. Brain Res. 171, 213-224.

Onoe, H., Komori, M., Onoe, K., Takechi, H., Tsukada, H., and Watanabe, Y.(2001). Cortical networks recruited for time perception: a monkey positron emission tomography (PET) study. Neuroimage 13, 37-45.

Pang, K. C., Yoder, R. M., and Olton, D. S. (2001). Neurons in the lateral agranular frontal cortex have divided attention correlates in a simultaneous temporal processing task. Neuroscience 103, 615-628.

Paxinos, G., and Watson, C. (1998). The Rat Brain in Stereotaxic Coordinates, 4th Edn. San Diego, Academic Press.
Pouthas, V., George, N., Poline, J. B., Pfeuty, M., Vandemoorteele, P. F., Hugueville, L., Ferrandez, A. M., Lehéricy, S., Lebihan, D., and Renault, B. (2005). Neural network involved in time perception: an fMRI study comparing long and short interval estimation. Human Brain Mapp. 25, 433-441.

Quintana, J., and Fuster,J.M. (1999). From perception to action: temporal integrative functions of prefrontal and parietal neurons. Cereb. Cortex 9, 213-221.

Rainer, G., Rao, S. C., and Miller, E. K. (1999). Prospective coding for objects in primate prefrontal cortex. J. Neurosci. 19, 5493-5505.

Rao,S.M.,Mayer,A.R.,andHarrington, D. L. (2001). The evolution of brain activation during temporal processing. Nat. Neurosci. 4, 317-323.

Rosenkilde, C. E., and Divac, I. (1976). Time-discrimination performance in cats with lesions in prefrontal cortex and caudate nucleus. J. Comp. Physiol. Psychol. 90, 343-352.

Rubia, K., Overmeyer, S., Taylor, E., Brammer, M., Williams, S., Simmons, A., Andrew, C., and Bullmore, E. (1998). Prefrontal involvement in "temporal bridging" and timing movement. Neuropsychologia 36, 1283-1293.

Stevens, M. C., Kiehl, K. A., Pearlson, G., and Calhoun, V.D. (2007). Functional neural circuits for mental timekeeping. Human Brain Mapp. 28, 394-408.

Tregellas, J. R., Davalos, D. B., and Rojas, D. C. (2006). Effect of task difficulty on the functional anatomy of temporal processing. Neuroimage 32, 307-315.

Conflict of Interest Statement: The authors declare that the research was conducted in the absence of any commercial or financial relationships that could be construed as a potential conflict of interest.

Received: 17 September 2009; paper pending published: 28 September 2009; accepted: 02 October 2009; published online: 03 November 2009.

Citation: Kim J, Jung AH, Byun J, Jo S and Jung $M W$ (2009) Inactivation of medial prefrontal cortex impairs time interval discrimination in rats. Front. Behav. Neurosci. 3:38. doi: 10.3389/neuro.08.038.2009

Copyright $\odot 2009$ Kim, Jung, Byun, Jo and Jung. This is an open-access article subject to an exclusive license agreement between the authors and the Frontiers Research Foundation, which permits unrestricted use, distribution, and reproduction in any medium, provided the original authors and source are credited. 